\title{
Spontaneous rupture of an incisional hernia
}

\author{
B.D. Dhaigude, MBBS MS¹, R.N. Bharadwaj, MBBS MS², Abhishek Kunal, MBBS ${ }^{3}$, \\ Shreyas Bhadranavar, MBBS$^{4}$, Manan Singh, MBBS $^{5}$, M. Vishwanath, MBBS 6 \\ 1 Associate Professor, Pad. Dr. D.Y Patil Medical College Pimpri, Pune, India. \\ 2 Professor, Pad. Dr. D.Y. Patil Medical College Pimpri, Pune, India. \\ 3 Resident, Pad. Dr. D.Y Patil Medical College Pimpri, Pune, India. \\ 4 Resident, Pad. Dr. D.Y Patil Medical College Pimpri, Pune, India. \\ 5. Resident, Pad. Dr. D.Y Patil Medical College Pimpri, Pune, India. \\ 6 Resident, Pad. Dr. D.Y Patil Medical College Pimpri, Pune, India.
}

Key words: Spontaneous rupture; Incisional hernia.

\section{Introduction}

An incisional hernia is represented by the protrusion of hollow viscera from their physiological position through an area of weakness in a surgical scar. Spontaneous rupture of abdominal herniae is very rare and usually occurs in incisional herniae [1] with only very few cases reported in literature. An incisional hernia is a frequently observed complication after abdominal surgery, with an incidence after midline laparotomy of 2 - 20\% [2]. We report a case of spontaneous rupture in a 45 year old female who had developed incisional hernia.

\section{Case report}

A 45 year old female presented to casualty with a ruptured incisional hernia following a fall from a bed. She had developed this hernia following hysterectomy complicated by wound infection at the age of 38. She was advised to undergo surgery in the past but refused.

On general examination she was afebrile with normal haemodynamic status. On examination of the abdomen a large irreducible incisional with depigmented, atrophic overlying skin and an ulcer through which omentum was protruding was observed. (Figure 1).

Figure 1. Omentum protruding through the defect in the hernial sac.

Correspondence: B.D. Dhaigude, Associate Professor Department of General Surgery Padmashree Dr. D.Y.Patil Medical College and Research Centre

Pimpri Pune - 18, India.

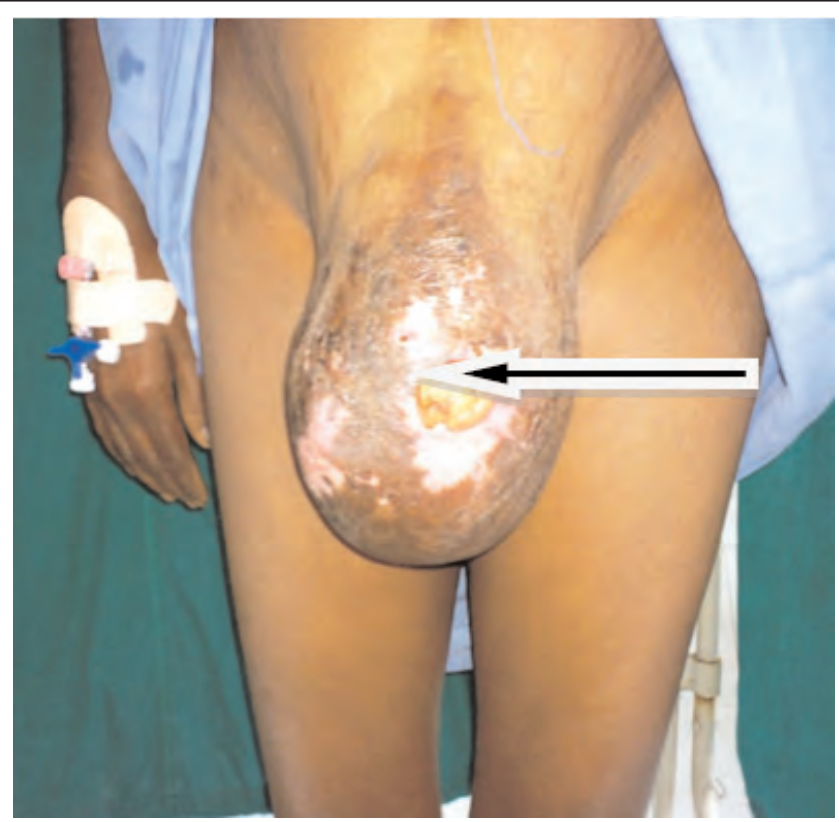

Routine imvestigations were normal. and emergency surgery was undertaken.

At operation a 6 x $10 \mathrm{~cm}$ defect was noted (Figure 2). This was enlarged, an omentectomy was done and bowel was returned to the peritoneal cavity after washing with normal saline. After removing all the atrophic avascular and scarred skin, anatomical repair of hernia was done. Closure was done with two free drains in the pelvis and paracolic gutter and a urethral catheter was maintained.

The post-operative period was uneventful and sutures were removed on the 10th post-operative day. After three months of follow up patient was asymptomatic.

Figure 2. Bowel and omentum protruding through the defect.

\section{Discussion}

Spontaneous rupture can occur with any type of abdominal 


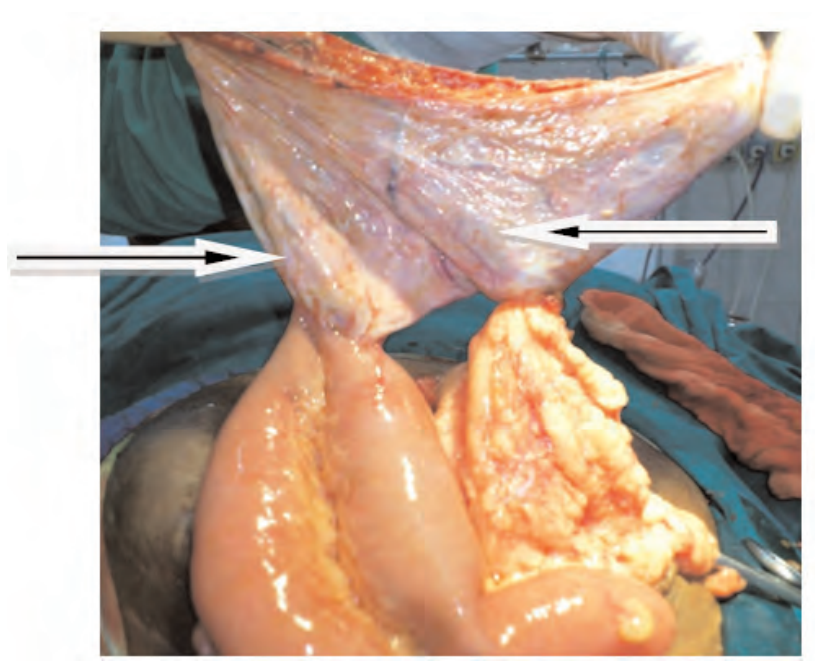

hernia but it is more commonly reported in incisional hernia [3]. Complications such as adhesions, incarceration of bowel and intestinal obstruction are well documented in association with incisional herniae but spontaneous rupture is very rarely reported in literature [4].

Delay in treatment increases the risk of rupture. The large hernia is contained only by its sac and thin atrophic avascular skin. Larger the hernia more atrophic and avascular is the overlying skin and this along with a thin sac leads to higher chances of rupture of incisional hernia.

The rupture may be sudden following any event which can increase the intra-abdominal pressure. Rupture of abdominal hernia demands emergency operation to prevent further obstruction, strangulation of bowel and to cover its contents.

In our patient the rupture of incisional hernia occurred because of sudden increase in intra-abdominal pressure due to abdominal trauma and thin atrophic avascular skin covering of the hernia. Delay in seeking prior surgical treatment was also a contributory factor in this case.
An anatomical repair was chosen over a mesh repair due to the exposure of the omentum to the exterior, surrounding soft tissue oedema and the danger of infection. Besides, the musculo aponeurotic structures around the hernia defect appeared adequately strong at surgery to allow an anatomical repair.

The drains and urethral catheter were left in situ to minimize the occurrence of postoperative intra-abdominal hypertension secondary to the replacement of a large volume of viscera intra-abdominally after the repair.

\section{Conclusion}

Spontaneous rupture of abdominal hernia is a very rare complication and it may occur in incisional herniae. The rupture of abdominal hernia demands emergency surgery. Timely surgical intervention can prevent this rare complication of incisional hernia.

\section{References}

1 Zinner MJ, Schwartz SI, Ellis H. Incisions, Closures, and management of the wound. Maingot's abdominal surgery. Appleton and Lange; 1997; 1(10): 423-2.

2. Nieuwenhuizen J, Halm JA, Jeekel J, Lange JF. The Netherlands, Natural course of incisional hernia and indication for repair. Scandinavian Journal of Surgery. 2007; 96(4): 293-6

PMid:18265856

3. Singla SL, Kalra U, Singh B, Naraula S, Dahiy P. Ruptured incisional hernia. Tropical Doctor 1997; 27 (2): 112-3.

PMID: 9133803

4. Aggarwal PK. Spontaneous rupture of incisional hernia. Br J Clin Pract. 1986; 40 (10): 443-4. 